\title{
ABC of Diabetes
}

\section{DIABETIC NEUROPATHY - II}

\section{Autonomic neuropathy}

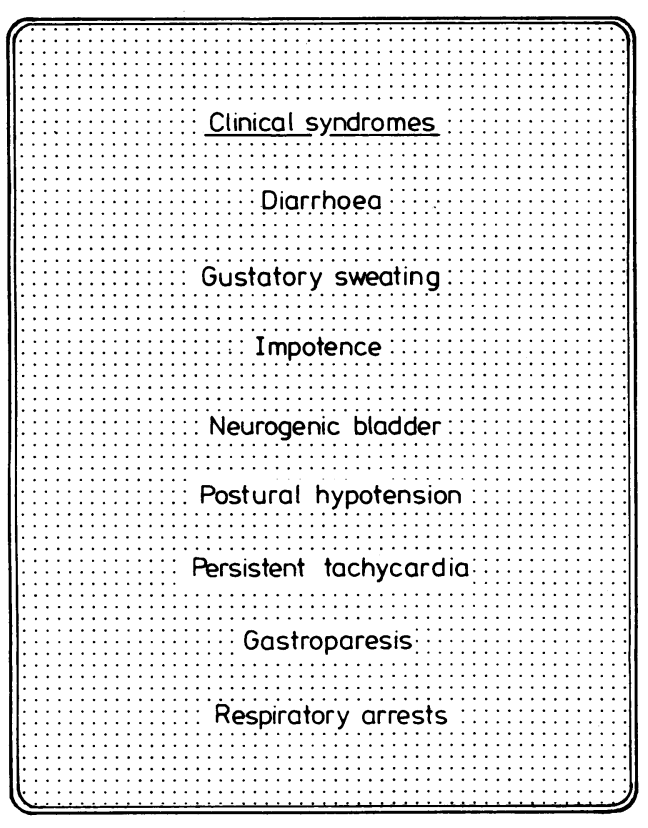

\section{Gastrointestinal system}

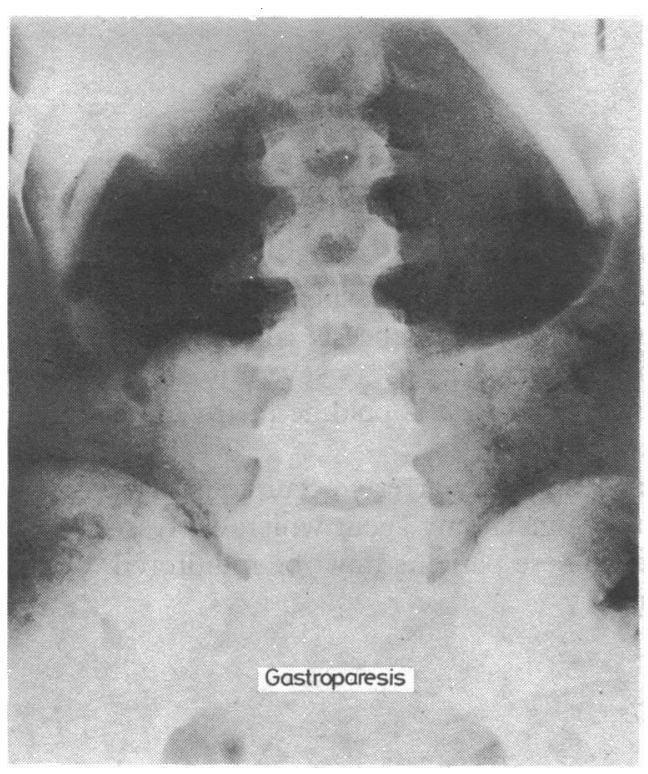

Diffuse damage to both parasympathetic and sympathetic nerves, probably developing in that order, is common in diabetics with diffuse peripheral neuropathy. Fortunately the disabling symptoms which result are not common, and even when they do occur some of them, especially diarrhoea, vomiting, and postural hypotension, are curiously intermittent.

Diarrhoea-This is a catastrophic watery diarrhoea with severe nocturnal exacerbations and faecal incontinence, preceded momentarily by characteristic abdominal rumblings. Malabsorption does not normally occur. The symptoms are intermittent, with normal bowel actions in between and sometimes even constipation. These features persist for months or years, sometimes eventually disappearing altogether. The diagnosis is made, firstly, by establishing the presence of peripheral and autonomic neuropathy and, secondly, by excluding other causes of diarrhoea such as coeliac disease. The diarrhoea may be treated with any antidiarrhoeal agent, the best of which is codeine phosphate. Tetracycline in two or three doses of $250 \mathrm{mg}$ has a dramatic effect in about half the cases. Tetracycline should be used only at the onset of an attack.

Gastroparesis-Diminished gastric motility and delayed stomach emptying occur sometimes in diabetics with autonomic neuropathy, but they rarely cause symptoms. Intermittent vomiting may occur, and in exceptional cases it may be intractable. Diagnosis is established by the presence of a gastric splash and screening during barium studies; and gastroscopy is needed to exclude other gastric disorders. Metoclopramide is useful in treatment: fortunately the episodes normally remit spontaneously. 


\section{Cardiovascular system}

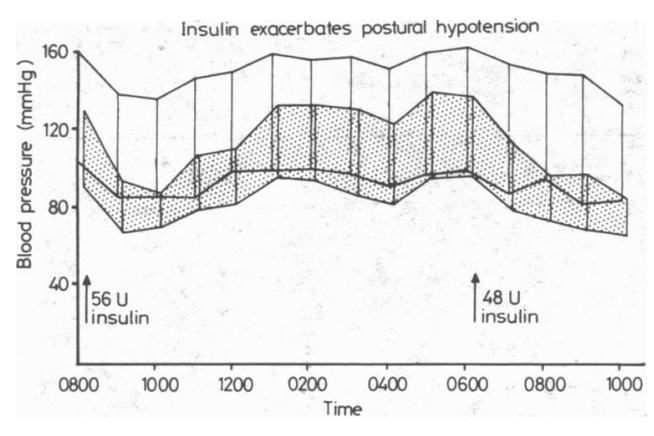

\section{Genitourinary system}
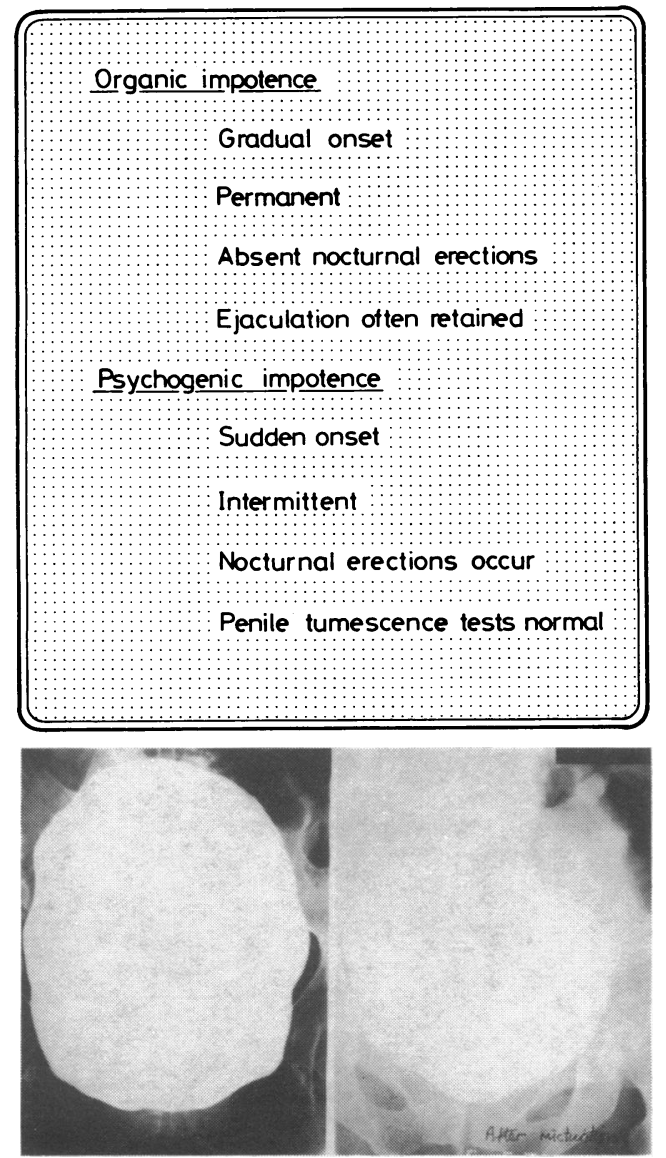

\section{Other effects}

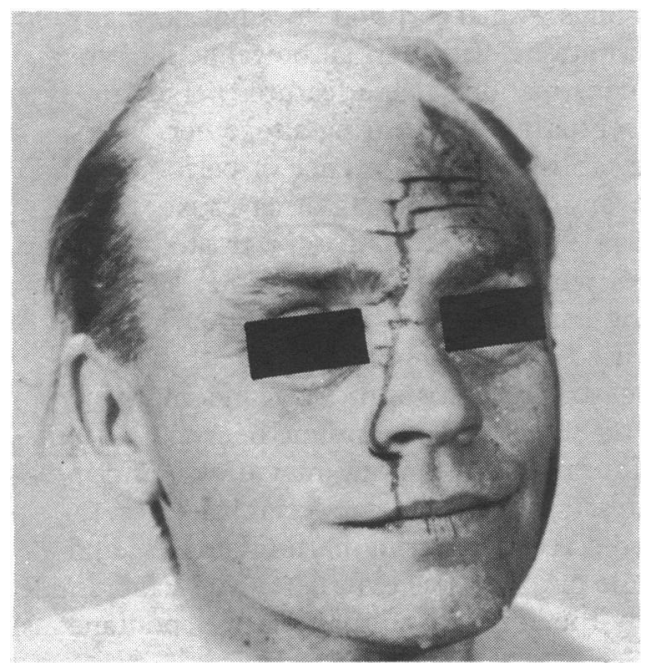

Postural hypotension (systolic pressure fall on standing of more than $30 \mathrm{~mm} \mathrm{Hg}$ ) needs treatment only if symptoms occur and if they are troublesome, which is rare. Patients should stop drugs which might aggravate hypotension (notably tranquillisers, antidepressants, and diuretics), sleep with the head of the bed raised, and wear full-length elastic stockings. The best results are obtained from measures which increase plasma volume-namely, a high salt intake and fludrocortisone (increasing the dose slowly to $0.4 \mathrm{mg}$ ). Failures are common, and the oedema which results from treatment may be disagreeable. Successful treatment has been reported with indomethacin; a combination of fludrocortisone, flurbiprofen, and ephedrine; the beta-blocker pindolol; and atrial tachypacing, but these reports have not been confirmed.

Impotence is a common problem among diabetics and non-diabetics, but it appears to be commoner in diabetics. Impotence is, however, most commonly due to psychogenic causes, and it is important that appropriate advice and treatment should be sought from trained counsellors.

Autonomic neuropathy causes erectile impotence, which is permanent and irreversible. Retrograde ejaculation can also occur. The onset of organic impotence in neuropathy is always gradi al, progressing over months or even years. Erectile ability fails first, ej ıculation declines later. Nocturnal erections are absent in these patients, whereas they are often retained in psychogenic impotence. It is often difficult to distinguish between organic and psychogenic impotence in diabetics. The presence of peripheral and autonomic neuropathy makes an organic cause more likely, especially when other autonomic symptoms are present. Newer techniques for examining nocturnal penile tumescence may help in diagnosis.

There is no cure for autonomic impotence. Hormone treatment with testosterone is useless because it serves only to increase libido without improving erectile ability. In many cases, careful explanation of the cause to the affected couples will allay their fears and anxieties. Sometimes an operation can be performed to insert a penile prosthesis. This can be successful because patients with erectile impotence often retain the ability to ejaculate.

Neurogenic bladder-Urinary retention is a serious and usually late complication of autonomic neuropathy. Apart from the discomfort, intractable urinary tract infections may develop. Treatment includes regular voiding helped by straining and abdominal pressure; the use of cholinergic drugs such as bethanechol; bladder-neck dilatation; and self-catheterisation. Careful resection of the bladder neck may help if other measures have failed. The illustration shows a grossly enlarged neurogenic bladder before and after micturation.

Gustatory sweating-Facial sweating (including scalp, neck, and shoulders) which occurs while eating tasty foods, notably cheese, is a common symptom of autonomic neuropathy. When it is very severe it can be treated with anticholinergic agents of which the best is poldine methylsulphate (Nacton).

Respiratory arrests - Transient respiratory arrests occur sometimes if susceptible neuropathic patients are given any agent which depresses respiration, notably anaesthetics. These patients must be monitored carefully even during minor surgery. 


\section{Diagnosis of autonomic neuropathy}

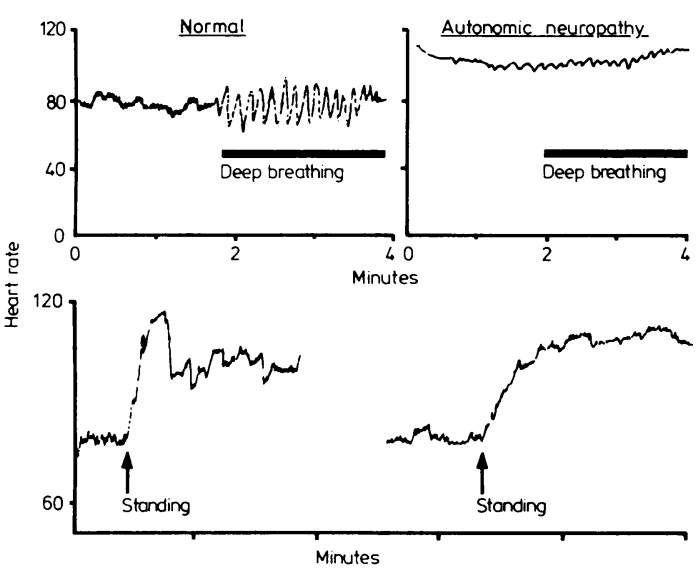

Gustatory sweating is the only symptom which is almost pathognomic of diabetic autonomic neuropathy. Peripheral neuropathy (at least absent ankle jerks) must be present before the diagnosis can be made. A resting tachycardia, postural hypotension, or a gastric splash may be present.

Loss of heart rate variability during deep breathing is the most reliable and simplest test of autonomic neuropathy. It is best assessed using a cardiotachograph during deep respirations ( 6 breaths per minute) taking average readings during 6 breaths; it can be performed using an ordinary electrocardiograph during a single deep breath ( 5 seconds in, 5 seconds out). The heart rate difference (maximum rate during inspiration minus minimum rate during expiration) in the under $55 \mathrm{~s}$ is always greater than 10. Heart rate increase on standing up should be greater than 12 at 15 seconds, and there should normally be an overshoot as well. The Valsalva manoeuvre can be included among the tests; a mercury sphygmomanometer is used, the patient blowing hard through the empty barrel of a $20 \mathrm{ml}$ syringe to maintain the mercury column at $40 \mathrm{~mm}$ for 10 seconds. Maximum heart rate during blowing, followed by minimum heart rate after cessation, are recorded. There should be a bradycardia after cessation of blowing; the ratio of maximum to minimum heart rate is normally greater than 1.21 and clearly abnormal when less than $1 \cdot 10$. Many other sophisticated tests need special equipment.

\section{Mononeuritis}

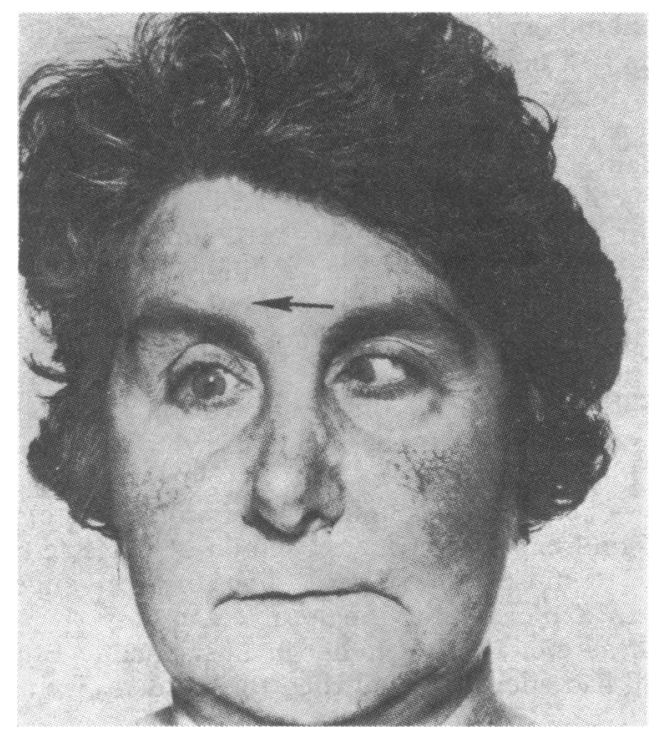

The rapid onset, severity, and eventual resolution of mononeuritis contrast sharply with the long-term nature and irreversibility of diffuse neuropathy. The two forms of neuropathy occur quite independently of each other.

Femoral neuropathy (diabetic amyotrophy)-Pain with or without wasting of one or both thighs is the cardinal feature of this disagreeable condition. The quality of the pain is similar to that in painful peripheral neuritis, and management is along similar lines. The knee jerk is absent, and sensation on the thigh may be altered or impaired. Other neurological disorders must be considered and excluded. Full recovery within about one year is the rule.

Radiculitis-Root involvement may cause the characteristic pain in almost any part of the body, notably the trunk.

Cranial nerve palsies-Third- and sixth-nerve palsies presenting with diplopia of sudden onset are characteristic. Pain behind the eye occurs sometimes in third-nerve palsies; the pupil is usually spared, and ptosis does not normally occur. Full examination and careful follow-up are needed, but extensive investigation is not normally required. Complete recovery occurs spontaneously in about three months.

Dr Peter J Watkins, MD, FRCP, is consultant physician, Diabetic Department, King's College Hospital, London.

The photograph of sixth nerve palsy was reproduced, with permission, from An Atlas of Clinical Neurology by John D Spillane, published by Oxford University Press.

\section{Necrobiosis lipoidica diabeticorum}

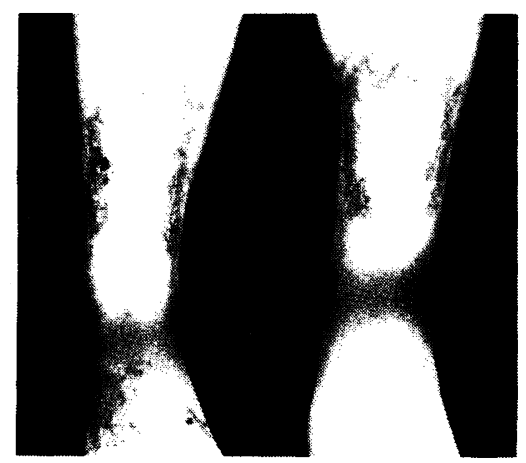

Necrobiosis is an uncommon and unsightly blemish of the skin which affects a few diabetic women. It is unrelated to microvascular complications. The shin is the most common site. The lesions show rather atrophic skin at the centre with obviously dilated capillaries (telangiectasis) and a slight raised pinkish rim; ulceration sometimes occurs. The lesions are indolent and rarely resolve. There is no effective treatment although steroid applications and even injections have been attempted. 